\title{
ERROR AND STABILITY ANALYSIS OF BOUNDARY METHODS FOR ELLIPTIC PROBLEMS WITH INTERFACES
}

\author{
ZI-CAI LI AND RUDOLF MATHON
}

\begin{abstract}
In boundary methods, piecewise particular solutions are employed to solve a given elliptic equation within subdomains of some region of interest. A boundary approximation is then obtained by satisfying the interior and exterior boundary conditions in a least squares sense. In this paper, we examine convergence, derive error norm bounds for approximate solutions and conduct a stability analysis of the associated algebraic problem. The aim of this analysis is to help choosing good partitions of subdomains. Finally, numerical experiments are carried out for a typical interface problem, demonstrating that very accurate solutions can be obtained while at the same time keeping small the condition numbers of the associated coefficient matrices.
\end{abstract}

\section{INTRODUCTION}

Consider an elliptic boundary value problem on a domain divided into several subdomains by artificial or material interfaces. If the admissible functions consist of particular solutions of the underlying elliptic equation on the subdomains, an approximate solution can be obtained by satisfying the exterior boundary conditions and the continuity conditions on the interior boundary as much as possible in a least squares sense. Since the approximation is performed only on the exterior and interior boundaries, we call these methods boundary methods (see Li [12] and Li, Mathon and Sermer [16]).

The advantages of boundary methods are summarized as follows:

1. It is easy to solve problerns with corners and interface singularities as well as with unbounded domains, with which the finite element and finite difference methods have difficulties coping.

2. The solution procedure is simple to carry out because only the interior and exterior boundary conditions are taken into account in the solution process.

3. A very accurate solution can be obtained by using relatively few expansion terms of particular solutions (approximation in one lower dimension), thus saving on work and storage space.

4. It is possible to estimate errors in the approximate solutions, although the exact solution of the physical problem is unknown. In this paper, a useful relation for the error behavior will be established,

$$
\text { (1.1) }\|\varepsilon\|_{H}=O\left(M|\varepsilon|_{B}\right) \text {, }
$$

Received May 16, 1988; revised October 28, 1988.

1980 Mathematics Subject Classification (1985 Revision). Primary 65N10, 65N30. larity.

Key words and phrases. Boundary methods, elliptic boundary value problem, interface, singu- 
where $M$ is the total number of unknown coefficients in the piecewise expansions used. The formula (1.1) is significant in practical calculation because we can compute the error in the domain $\|\varepsilon\|_{H}$ in terms of the errors on the boundary $|\varepsilon|_{B}$, which is naturally obtained from the boundary methods. Once the errors of solutions are known, we can easily control the calculation procedure.

However, the following two difficulties arise in using boundary methods.

1. Piecewise particular solutions of elliptic equations have to be known. For the most important elliptic equations in application, we may find useful particular solutions in textbooks of partial differential equations, e.g., Tikhonov and Samarskii [21]. But quite often, an analysis is essential to yield asymptotic expansions near singular points and infinity, since the behavior of the solution near them is unknown or unclear.

2. Stability of numerical solutions is also important. The numerical experiments in [16] report that using several subdomains leads to better stability but lower accuracy of the numerical solutions. In fact, stability will rely substantially on both the choice of piecewise particular solutions and the partition of the solution domain. Our intention in this paper is to use a stability analysis to guide us in choosing partitions so that better solutions can be obtained via boundary methods.

In this paper we present an analysis of errors and stability, as well as numerical experiments for the equation

$$
-\Delta u+u=0,
$$

with interface singularities by boundary methods on a decomposed domain, to show not only that the solutions obtained are extremely accurate, but also that the condition numbers of the coefficient matrices are surprisingly small.

It should be noted that the infinite grid refinements of Han [8] and Thatcher $[19,20]$ cannot be applied to the equation (1.2).

\section{BOUNDARY APPROXIMATIONS}

Let $\Omega$ be a bounded domain divided by a surface $\Gamma_{0}$ into two subdomains $\Omega^{+}$and $\Omega^{-}$, i.e., $\Omega=\Omega^{+} \cup \Omega^{-}$. In this paper, we will consider the piecewise equations

$$
\begin{array}{ll}
p^{+}(-\Delta u+u)=0 & \text { in } \Omega^{+}, \\
p^{-}(-\Delta u+u)=0 & \text { in } \Omega^{-},
\end{array}
$$

with the interior and exterior boundary conditions

$$
\begin{aligned}
& u^{+}=u^{-}, \quad p^{+} u_{\nu}^{+}=p^{-} u_{\nu}^{-} \quad \text { on } \Gamma_{0}, \\
& u=f \quad \text { on } \Gamma_{D}, \quad u_{\nu}=g \quad \text { on } \Gamma_{N},
\end{aligned}
$$

where $f$ and $g$ are sufficiently smooth functions, $\Gamma_{D} \cup \Gamma_{N}=\partial \Omega$ and $\Gamma_{D} \neq \varnothing$, $u_{\nu}$ is the normal derivative, and $u^{ \pm}=u$ in $\Omega^{ \pm}$.

Let $p^{+}$and $p^{-}$be different positive constants that are related to different materials in $\Omega^{+}$and $\Omega^{-}$, i.e.,

$$
p^{+} \neq p^{-}
$$


Then equations (2.1) and (2.2) become

$$
-\Delta u^{ \pm}+u^{ \pm}=0 \text { in } \Omega^{ \pm} .
$$

When $p^{+}=p^{-}, \Gamma_{0}$ is an artificial interface, cf. [16].

We need some notation. Define a space on $\Omega$,

$$
\begin{aligned}
H=\left\{v \in L_{2}(\Omega) \mid v \in H^{1}\left(\Omega^{+}\right),\right. & v \in H^{1}\left(\Omega^{-}\right), \\
& \text {and } \left.-\Delta v+v=0 \text { in } \Omega^{+} \text {and } \Omega^{-}\right\},
\end{aligned}
$$

and a functional on $\Gamma_{0}$ and $\partial \Omega$,

$$
\begin{aligned}
I(v)= & \int_{\Gamma_{D}}(v-f)^{2} d s+w^{2} \int_{\Gamma_{N}}\left(v_{\nu}-g\right)^{2} d s+\int_{\Gamma_{0}}\left(v^{+}-v^{-}\right)^{2} d s \\
& +w^{2} \int_{\Gamma_{0}}\left(p^{+} v_{\nu}^{+}-p^{-} v_{\nu}^{-}\right)^{2} d s,
\end{aligned}
$$

where $w$ is also a positive weight. On $H \times H$ we will use a bilinear form $[\cdot, \cdot]$ defined by

$$
\begin{aligned}
{[u, v]=} & \int_{\Gamma_{D}} u v d s+w^{2} \int_{\Gamma_{N}} u_{\nu} v_{\nu} d s+\int_{\Gamma_{0}}\left(u^{+}-u^{-}\right)\left(v^{+}-v^{-}\right) d s \\
& +w^{2} \int_{\Gamma_{0}}\left(p^{+} u_{\nu}^{+}-p^{-} u_{\nu}^{-}\right)\left(p^{+} v_{\nu}^{+}-p^{-} v_{\nu}^{-}\right) d s
\end{aligned}
$$

and an inner product

$$
|v|_{B}^{2}=[v, v]
$$

The norm $\|v\|_{H}$ on $H$ is defined as follows:

$$
\|v\|_{H}=\left\{\|v\|_{1, \Omega^{+}}^{2}+\|v\|_{1, \Omega^{-}}^{2}\right\}^{1 / 2}
$$

where the Sobolev norms are

$$
\|v\|_{m, \Omega}=\left\{\sum_{|\alpha| \leq m} \int_{\Omega}\left|D^{\alpha} v\right|^{2} d x\right\}^{1 / 2} .
$$

Consider finite-dimensional spaces $S_{m, n} \subseteq H$ such that

$$
\begin{aligned}
& S_{m, n}=\left\{v \mid v=v^{+}=\sum_{i=1}^{m} c_{i} \psi_{i}^{+} \text {on } \Omega^{+},\right. \\
& \left.\quad \text { and } v=v^{-}=\sum_{i=1}^{n} d_{i} \psi_{i}^{-} \text {on } \Omega^{-}\right\},
\end{aligned}
$$

where $\psi_{i}^{ \pm}$are complete sets of particular solutions of (2.1) and (2.2). A boundary approximation, $u_{m, n} \in S_{m, n}$, is then found by

$$
I\left(u_{m, n}\right)=\min _{v \in S_{m, n}} I(v) .
$$


It is worth pointing out that the boundary methods here may fall into the class of general, weighted least squares methods for elliptic systems of Aziz, Kellogg and Stephens [3], which are applied both within small elements (viewed as subdomains here) and on their boundaries (viewed as interfaces). Since only particular solutions are chosen to be admissible functions, the number of unknown coefficients decreases drastically. Good boundary approximation can be obtained even if several subdomains are used (see the numerical examples in $\S 5)$. It would also be interesting to develop least squares methods in which the number of trial functions (as in this paper) and the number of subdomains (as in [3] and in the finite element method [18]) are both changing, in particular for those elliptic problems for which particular solutions cannot be found. In this latter case, also residuals within the subdomains (as in [3]) have to be considered.

\section{ERROR ANALYSIS}

For the space $S_{m, n}$, we assume that the inverse property and the approximability property hold as in [16].

Inverse Property. For any $v \in S_{m, n}$ we have

$$
\left|v_{\nu}\right|_{0, \Gamma_{D}} \leq k_{m, n}\|v\|_{H}, \quad\left|v_{\nu}^{+}\right|_{0, \Gamma_{0}} \leq k_{m, n}\|v\|_{H}
$$

where $k_{m, n}$ is unbounded as $m, n \rightarrow \infty$ and the norms on the boundary or interface are defined by

$$
|v|_{0, \Gamma}=\left(\int_{\Gamma} v^{2} d s\right)^{1 / 2}
$$

We note that in (3.1), the inverse property on the interface $\Gamma_{0}$ is assumed only for $\Gamma_{0}^{+}$, because the norm bounds of errors in the following proof are needed only on one side of $\Gamma_{0}$, say $\Gamma_{0}^{+}($see $(3.11))$.

Approximability Property. For any $u \in H$, there exists a function $v \in S_{m, n}$ such that

$$
\begin{array}{cc}
\left\|u^{+}-v^{+}\right\|_{l, \partial \Omega^{+}} \leq \alpha_{n, k, l}^{+}\left|u^{+}\right|_{k, \partial \Omega^{+}}, & 0 \leq l \leq k, \\
\left\|u^{-}-v^{-}\right\|_{l, \partial \Omega^{-}} \leq \alpha_{m, q, l}^{-}\left|u^{-}\right|_{q, \partial \Omega^{-}}, & 0 \leq l \leq q,
\end{array}
$$

where $\alpha_{n, k, l}^{+}, \alpha_{m, q, l}^{-} \rightarrow 0$ as $m, n \rightarrow \infty$, and

$$
\|v\|_{l, \partial \Omega}=\left\{\sum_{|\alpha| \leq l} \int_{\partial \Omega}\left(\frac{\partial^{\alpha} v}{\partial s^{\alpha}}\right) d s\right\}^{1 / 2}, \quad|v|_{l, \partial \Omega}=\left\{\sum_{|\alpha|=l} \int_{\partial \Omega}\left(\frac{\partial^{\alpha} v}{\partial s^{\alpha}}\right) d s\right\}^{1 / 2} .
$$

Approximability properties may be found in Cheney [5] for some spaces, or in Eisenstat [6] for the Bergman-Vekua space. For the equation $-\Delta u+u=0$, the approximability properties of $S_{m, n}$ can be obtained only when the given subdomains are imbedded into sectors of a circle, which are within the solution domains. In other cases, a further study of the approximating spaces needs to 
be done (refer to the density study in Aziz, Dorr and Kellogg [2] and Browder [4]).

Under the above assumptions, we will provide error estimates and establish the relation (1.1). First we cite two lemmas and a theorem, which can be proved along the same lines as in [16].

For $v \in H$, there exists an imbedding constant $C_{s}>0$ in the Sobolev imbedding theorem such that [17]

$$
|v|_{0, \Gamma_{D}} \leq C_{s}\|v\|_{H}, \quad\left|v^{ \pm}\right|_{0, \Gamma_{0}} \leq C_{s}\|v\|_{1, \Omega^{ \pm}} \leq C_{s}\|v\|_{H} .
$$

Then we have

Lemma 3.1. Let $v \in S_{m, n}$, and suppose that the inverse property holds. Then, when $w>0$, there exist norm bounds

$$
\|v\|_{H} \leq t\left(k_{m, n}+\frac{C_{s}}{w}\right)|v|_{B}
$$

where the constant

$$
t=\frac{\max \left(p^{+}, p^{-}\right)}{\min \left(p^{+}, p^{-}\right)} .
$$

Lemma 3.2. Let $u$ be the solution of equations (2.1)-(2.4). Then for any $w>$ 0 , there exists a unique function, $u_{m, n} \in S_{m, n}$, such that

$$
\left[u_{m, n}, v\right]=\int_{\Gamma_{D}} f v d s+w^{2} \int_{\Gamma_{N}} g v_{\nu} d s \quad \text { for all } v \in S_{m, n},
$$

$$
\begin{gathered}
{\left[u-u_{m, n}, v\right]=0 \text { for all } v \in S_{m, n},} \\
\left\|u_{m, n}\right\|_{H} \leq t\left(k_{m, n}+\frac{C_{s}}{w}\right)\left\{|f|_{0, \Gamma_{D}}+w|g|_{0, \Gamma_{N}}\right\} .
\end{gathered}
$$

Also, $u_{m, n}$ minimizes I over $S_{m, n}$ if and only if (3.5) holds.

The next estimate bounds the errors in the $H$-norm.

Theorem 3.1. Let $u \in H^{1}(\Omega)$ be the solution of (2.1)-(2.4), and let $u_{m, n} \in$ $S_{m, n}$ be the boundary approximation (3.5). If the inverse property holds, then for any $w>0$, there exist error bounds

$$
\left\|u-u_{m, n}\right\|_{H} \leq \inf _{v \in S_{m, n}}\left\{\|u-v\|_{H}+t\left(k_{m, n}+\frac{C_{s}}{w}\right)|u-v|_{B}\right\},
$$

where $t$ is defined in Lemma 3.1, $k_{m, n}$ is defined in (3.1) and $C_{s}$ is given in (3.3)

Corollaries similar to those in [16] can be easily derived. Here, our main interest is in the relation between $\left\|u-u_{m, n}\right\|_{H}$ and $\left|u-u_{m, n}\right|_{B}$ only. 
Let $u$ be the solution of $(2.1)-(2.4)$; then the error norms satisfy

$$
\begin{aligned}
|\varepsilon|_{B}^{2}= & \left|u-u_{m, n}\right|_{B}^{2} \\
= & \int_{\Gamma_{D}}\left(u_{m, n}-f\right)^{2} d s+w^{2} \int_{\Gamma_{N}}\left(\frac{\partial u_{m, n}}{\partial \nu}-g\right)^{2} d s \\
& +\int_{\Gamma_{0}}\left(u_{m, n}^{+}-u_{m, n}^{-}\right)^{2} d s \\
& +w^{2} \int_{\Gamma_{0}}\left(p^{+} \frac{\partial u_{m, n}^{+}}{\partial \nu}-p^{-\frac{\partial u_{m, n}^{-}}{\partial \nu}}\right)^{2} d s .
\end{aligned}
$$

We note that the true solution $u$ disappears in $|\varepsilon|_{B}$ (see (3.9)) and also the values of $|\varepsilon|_{B}$ are easily computed in the least squares procedure employed in the boundary method. We can then evaluate $\|\varepsilon\|_{H}$ in terms of $|\varepsilon|_{B}$. Such a relation for the norms is given in the following theorem.

Theorem 3.2. Let $u \in H^{1}(\Omega)$ be the solution of (2.1)-(2.4), and let $u_{m, n} \in$ $S_{m, n}$ be the boundary approximation (3.5). If the inverse property holds for the difference $u-u_{m, n}$, then for any $w>0$, the error is bounded by

$$
\left\|u-u_{m, n}\right\|_{H} \leq t\left(k_{m, n}+\frac{C_{s}}{w}\right)\left|u-u_{m, n}\right|_{B}
$$

where the constants $t, k_{m, n}$ and $C_{s}$ are the same as in Theorem 3.1 .

Proof. Letting $v=u_{m, n} \in S_{m, n}$ and $\delta=\min \left(p^{+}, p^{-}\right)$, we have from (2.3)

$$
\begin{aligned}
&\|u-v\|_{H}^{2} \leq \frac{1}{\delta} \mid \int_{\partial \Omega^{+}} p^{+}\left(u_{\nu}^{+}-v_{\nu}^{+}\right)\left(u^{+}-v^{+}\right) d s \\
&+\int_{\partial \Omega^{-}} p^{-}\left(u_{v}^{-}-v_{\nu}^{-}\right)\left(u^{-}-v^{-}\right) d s \mid \\
& \leq \frac{1}{\delta}\left\{\left|\int_{\Gamma_{D}} p\left(u_{\nu}-v_{\nu}\right)(f-v) d s\right|+\left|\int_{\Gamma_{N}} p\left(g-v_{\nu}\right)(u-v) d s\right|\right. \\
&+\mid \int_{\Gamma_{0}}\left[\left(v^{+}-v^{-}\right) p^{+}\left(u_{\nu}^{+}-v_{\nu}^{+}\right)\right. \\
& \leq t\left\{\left|u_{\nu}-v_{\nu}\right|_{0, \Gamma_{D}}|f-v|_{0, \Gamma_{D}}+\left|g-v_{\nu}\right|_{0, \Gamma_{N}}|u-v|_{0, \Gamma_{N}}\right. \\
&+\left|v^{+}-v^{-}\right|_{0, \Gamma_{0}}\left|u_{\nu}^{+}-v_{\nu}^{+}\right|_{0, \Gamma_{0}} \\
&\left.\quad+\left|u^{-}-v^{-}\right|_{0, \Gamma_{0}}\left|p^{+} v_{\nu}^{+}-p^{-} v_{\nu}^{-}\right|_{0, \Gamma_{0}}\right\}
\end{aligned}
$$

The inverse property (3.1) and the imbedding theorem (3.3) imply that

$$
\begin{aligned}
\|u-v\|_{H}^{2} \leq t & \left\{k_{m, n}|f-v|_{0, \Gamma_{D}}+C_{s}\left|g-v_{\nu}\right|_{0, \Gamma_{N}}\right. \\
& \left.+k_{m, n}\left|v^{+}-v^{-}\right|_{0, \Gamma_{0}}+C_{s}\left|p^{+} v_{\nu}^{+}-p^{-} v_{\nu}^{-}\right|_{0, \Gamma_{0}}\right\}\|u-v\|_{H} .
\end{aligned}
$$


Dividing both sides by $\|u-v\|_{H}$ and using the definition (2.10), we obtain

$$
\|u-v\|_{H} \leq t\left(k_{m, n}+\frac{C_{s}}{w}\right)|u-v|_{B}
$$

The inequality (3.10) follows from the substitution $v=u_{m, n}$, thus completing the proof of Theorem 3.2.

Based on Theorem 3.2, we have

Corollary 3.1. Let the weight $w=1 / M$, where $M=\max (m, n)$, and suppose that all conditions in Theorem 3.2 hold. Also suppose that the constant $k_{m, n}$ in the inverse property satisfies

$$
k_{m, n} \leq C M,
$$

where $C$ is a bounded constant independent of $m, n$ and $u$. Then

$$
\left\|u-u_{m, n}\right\|_{H}=O\left(M\left|u-u_{m, n}\right|_{B}\right) \text {. }
$$

A discussion of the bounds on $k_{m, n}$ can be found in [12].

\section{Stability analysis}

In this section we will present a stability analysis for boundary methods based on domain decomposition and discuss the choice of geometric shapes for the subdomains.

In order to discuss stability of the solution $u_{m, n}$, we need to estimate the value of the condition numbers

$$
\text { cond }=\left(\frac{\lambda_{\max }(B)}{\lambda_{\min }(B)}\right)^{1 / 2} .
$$

Here, $\lambda_{\max }$ and $\lambda_{\min }$ are the maximal and minimal eigenvalues of the associated coefficient matrix $B$ defined by

$$
\vec{X}^{T} B \vec{X}=|v|_{B}^{2}, \quad v \in S_{m, n},
$$

where the vector $\vec{X}$ is composed of the unknown coefficients $c_{i}$ and $d_{i}$ in (2.12).

Let the bounded domains $\underline{\Omega}^{ \pm}$and $\bar{\Omega}^{ \pm}$be such that

$$
\underline{\Omega}^{+} \subseteq \Omega^{+} \subseteq \bar{\Omega}^{+}, \quad \underline{\Omega}^{-} \subseteq \Omega^{-} \subseteq \bar{\Omega}^{-},
$$

and define two matrices as follows:

$$
F_{\Omega^{+}}=\left(f_{i, j}^{+}\right), \quad F_{\Omega^{-}}=\left(f_{i, j}^{-}\right),
$$

where the matrix elements $f_{i, j}^{ \pm}$are

$$
\begin{gathered}
f_{i, j}^{+}=\left(\psi_{i}^{+}, \psi_{j}^{+}\right)_{\Omega^{+}}+\left(\frac{\partial \psi_{i}^{+}}{\partial x}, \frac{\partial \psi_{j}^{+}}{\partial x}\right)_{\Omega^{+}}+\left(\frac{\partial \psi_{i}^{+}}{\partial y}, \frac{\partial \psi_{j}^{+}}{\partial y}\right)_{\Omega^{+}}, \\
f_{i, j}^{-}=\left(\psi_{i}^{-}, \psi_{j}^{-}\right)_{\Omega^{-}}+\left(\frac{\partial \psi_{i}^{-}}{\partial x}, \frac{\partial \psi_{j}^{-}}{\partial x}\right)_{\Omega^{-}}+\left(\frac{\partial \psi_{i}^{-}}{\partial y}, \frac{\partial \psi_{j}^{-}}{\partial y}\right)_{\Omega^{-}} .
\end{gathered}
$$


Then we have

$$
\left(\vec{X}^{+}\right)^{T} F_{\Omega^{+}} \vec{X}^{+}=\left\|v^{+}\right\|_{1, \Omega^{+}}^{2}, \quad\left(\vec{X}^{-}\right)^{T} F_{\Omega^{-}} \vec{X}^{-}=\left\|v^{-}\right\|_{1, \Omega^{-}}^{2}
$$

where

$$
\begin{gathered}
v^{+}=\sum_{i=1}^{m} c_{i} \psi_{i}^{+}, \quad v^{-}=\sum_{i=1}^{n} d_{i} \psi_{i}^{-} \\
\vec{X}^{+}=\left(c_{1}, c_{2}, \ldots, c_{m}\right)^{T}, \\
\vec{X}^{-}=\left(d_{1}, d_{2}, \ldots, d_{n}\right)^{T} .
\end{gathered}
$$

We will prove the following results.

Theorem 4.1. Suppose that for any $v \in S_{m, n}$,

$$
\left|v_{\nu}\right|_{0, \Gamma_{N}} \text { and }\left|v_{\nu}^{ \pm}\right|_{0, \Gamma_{0}} \leq k_{m, n}\|v\|_{H}
$$

for some positive constants $k_{m, n}$. Then for any $w>0$, there exist bounds

(4.10) cond $\leq t w\left[\left(1+p_{\max }\right) k_{m, n}+\frac{C_{s}}{w}\right]^{2}\left\{\frac{\max \left[\lambda_{\max }\left(F_{\bar{\Omega}^{+}}\right), \lambda_{\max }\left(F_{\bar{\Omega}^{-}}\right)\right]}{\min \left[\lambda_{\min }\left(F_{\underline{\Omega}^{+}}\right), \lambda_{\min }\left(F_{\underline{\Omega}^{-}}\right)\right]}\right\}^{1 / 2}$,

where $p_{\max }=\max \left(p^{+}, p^{-}\right), C_{s}$ is defined in (3.3), and $t$ is given in Lemma 3.1 .

Proof. We have from Lemma 3.1

$$
|v|_{B}^{2} \geq \frac{\|v\|_{H}^{2}}{t^{2}\left(k_{m, n}+C_{s} / w\right)^{2}} \geq \frac{\left\|v^{+}\right\|_{1, \underline{\Omega}^{+}}^{2}+\left\|v^{-}\right\|_{1, \underline{\Omega}^{-}}^{2}}{t^{2}\left(k_{m, n}+C_{s} / w\right)^{2}}
$$

Then

$$
\lambda_{\min }(B)=\min \frac{|v|_{B}^{2}}{\vec{X}^{T} \vec{X}} \geq \frac{1}{t^{2}\left(k_{m, n}+C_{s} / w\right)^{2}} \min \frac{\left\|v^{+}\right\|_{1, \Omega^{+}}^{2}+\left\|v^{-}\right\|_{1, \underline{\Omega}^{-}}^{2}}{\vec{X}^{T} \vec{X}} .
$$

If we denote

$$
T=\left[\begin{array}{cc}
F_{\Omega^{+}} & 0 \\
0 & F_{\Omega^{-}}
\end{array}\right]
$$

we obtain a relation for the smallest eigenvalues of the matrices $T, F_{\underline{\Omega}^{+}}$and $F_{{\underline{\Omega^{-}}}^{-}}$

$$
\lambda_{\min }(T)=\min \frac{\left\|v^{+}\right\|_{1, \underline{\Omega}^{+}}^{2}+\left\|v^{-}\right\|_{1, \underline{\Omega}^{-}}^{2}}{\vec{X}^{T} \vec{X}}=\min \left[\lambda_{\min }\left(F_{\underline{\Omega}^{+}}\right), \lambda_{\min }\left(F_{\underline{\Omega}^{-}}\right)\right] .
$$

Obviously,

$$
\lambda_{\min }(B) \geq \frac{1}{t^{2}\left(k_{m, n}+C_{s} / w\right)^{2}} \min \left[\lambda_{\min }\left(F_{\underline{\Omega}^{+}}\right), \lambda_{\text {min }}\left(F_{\underline{\Omega}^{-}}\right)\right] .
$$

Similarly, from the assumption (4.9) and the Sobolev imbedding theorem we can see that

$$
\begin{aligned}
|v|_{B}^{2} & \leq\left(C_{s}+p_{\max } w k_{m, n}\right)^{2}\|v\|_{H}^{2} \\
& \leq\left(C_{s}+p_{\max } w k_{m, n}\right)^{2}\left[\left\|v^{+}\right\|_{1, \bar{\Omega}^{+}}^{2}+\left\|v^{-}\right\|_{1, \bar{\Omega}^{-}}^{2}\right]
\end{aligned}
$$


Therefore,

$$
\lambda_{\max }(B) \leq\left(C_{s}+p_{\max } w k_{m, n}\right)^{2} \max \left[\lambda_{\max }\left(F_{\bar{\Omega}^{+}}\right), \lambda_{\max }\left(F_{\bar{\Omega}^{-}}\right)\right]
$$

It follows by computing (4.13) and (4.15) that

$$
\frac{\lambda_{\max }(B)}{\lambda_{\min }(B)} \leq t^{2} w^{2}\left[\left(1+p_{\max }\right) k_{m, n}+\frac{C_{s}}{w}\right]^{4} \frac{\max \left[\lambda_{\max }\left(F_{\bar{\Omega}^{+}}\right), \lambda_{\max }\left(F_{\bar{\Omega}^{-}}\right)\right]}{\min \left[\lambda_{\min }\left(F_{\underline{\Omega}^{+}}\right), \lambda_{\min }\left(F_{\underline{\Omega}^{-}}\right)\right]},
$$

which leads to the inequality (4.10). This completes the proof of Theorem 4.1.

Below, we will apply Theorem 4.1 to the partition displayed in Figure 1 and the admissible functions:

$$
\begin{array}{ll}
v^{+}=\sum_{i=1}^{m} c_{i} \frac{I_{\mu_{i}^{+}}(r)}{I_{\mu_{i}^{+}}\left(R_{0}^{+}\right)} \sin \mu_{i}^{+} \theta, \quad(r, \theta) \in \Omega^{+}, \\
v^{-}=\sum_{i=1}^{n} d_{i} \frac{I_{\mu_{i}^{-}}(\rho)}{I_{\mu_{i}^{-}}\left(R_{0}^{-}\right)} \sin \mu_{i}^{-} \phi, \quad(\rho, \phi) \in \Omega^{-},
\end{array}
$$

where $c_{i}$ and $d_{i}$ are known coefficients, $\mu_{i}^{ \pm}=i \pi / \Theta^{ \pm}, \Theta^{ \pm}$are the intersection angles, $(r, \theta)$ and $(\rho, \phi)$ are the polar coordinates with the origins $A$ and $A^{*}$, respectively, and the radii $R_{0}^{ \pm}$are defined by the formulas (4.19) below.

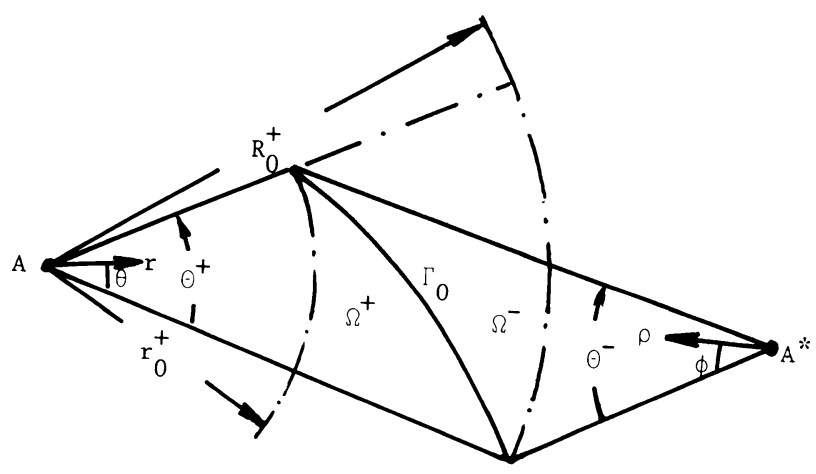

FIgURE 1. A partition of $\Omega=\Omega^{+} \cup \Omega^{-}$

Let the sectors $\bar{\Omega}^{ \pm}$and $\underline{\Omega}^{ \pm}$satisfy $(4.3)$ and

$$
\begin{aligned}
& \bar{\Omega}^{-}=\left\{0<\rho<R_{0}^{-} \text {and } 0<\phi<\Theta^{-}\right\}, \\
& \underline{\Omega}^{-}=\left\{0<\rho<r_{0}^{-} \text {and } 0<\phi<\Theta^{-}\right\}, \\
& \bar{\Omega}^{+}=\left\{0<r<R_{0}^{+} \text {and } 0<\theta<\Theta^{+}\right\}, \\
& \underline{\Omega}^{+}=\left\{0<r<r_{0}^{+} \text {and } 0<\theta<\Theta^{+}\right\} .
\end{aligned}
$$


In (4.17) and (4.18), $I_{\mu}(r)$ are the Bessel functions for a purely imaginary argument defined by (see Tikhonov and Samarskii [21])

$$
I_{\mu}(r)=\sum_{k=0}^{\infty} \frac{1}{\Gamma(k+1) \Gamma(k+\mu+1)}\left\{\frac{r}{2}\right\}^{2 k+\mu} .
$$

Then we have

Corollary 4.1. Let $v^{ \pm}$be admissible functions given by (4.17) and (4.18) on the division in Figure 1. If $m=O(n)$ and the conditions in Theorem 4.1 are satisfied, then there exists a bounded constant $C$ independent of $m, n$ and $u$ such that

(4.20) cond $\leq C t w\left[\left(1+p_{\max }\right) k_{m, n}+\frac{C_{s}}{w}\right]^{2} \max \left\{\left[\frac{R_{0}^{+}}{r_{0}^{+}}\right]^{\mu_{m}^{+}},\left[\frac{R_{0}^{-}}{r_{0}^{-}}\right]^{\mu_{n}^{-}}\right\}$,

where the radii $R_{0}^{ \pm}$and $r_{0}^{ \pm}$are defined by (4.19).

Proof. Using the orthogonality of $\sin \mu_{i}^{+} \theta$, we can see that

$$
\begin{aligned}
& \|v\|_{1, \bar{\Omega}^{+}}^{2}=\frac{\Theta^{+}}{2} \sum_{i=0}^{m} c_{i}^{2} \int_{0}^{R_{0}^{+}} r G_{i}^{+}(r) d r, \\
& \|v\|_{1, \underline{\Omega}^{+}}^{2}=\frac{\Theta^{+}}{2} \sum_{i=0}^{m} c_{i}^{2} \int_{0}^{r_{0}^{+}} r G_{i}^{+}(r) d r,
\end{aligned}
$$

where

$$
G_{i}^{+}(r)=\frac{\left[\left[I_{\mu_{i}^{+}}^{\prime}(r)\right]^{2}+\frac{\left(\mu_{i}^{+}\right)^{2}}{r^{2}} I_{\mu_{i}^{+}}^{2}(r)+I_{\mu_{i}^{+}}^{2}(r)\right]}{I_{\mu_{i}^{+}}^{2}\left(R_{0}^{+}\right)} .
$$

On the other hand, the functions $I_{\mu}(r)$ can also be expressed as (Abramowitz and Stegun [1, equation 9.6.18])

$$
I_{\mu}(r)=\frac{\left(\frac{1}{2} r\right)^{\mu}}{\Gamma\left(\frac{1}{2}\right) \Gamma\left(\mu+\frac{1}{2}\right)} \int_{-1}^{1} e^{ \pm r t}\left(1-t^{2}\right)^{\mu-1 / 2} d t .
$$

The Bessel functions $I_{\mu}(r)$ are known to satisfy the bounds

$$
\beta_{\mu} r^{\mu} e^{-\max r} \leq I_{\mu}(r) \leq \beta_{\mu} r^{\mu} e^{\max r},
$$

where

$$
\beta_{\mu}=\frac{\left(\frac{1}{2}\right)^{\mu}}{\Gamma\left(\frac{1}{2}\right) \Gamma\left(\mu+\frac{1}{2}\right)} \int_{-1}^{1}\left(1-t^{2}\right)^{\mu-1 / 2} d t,
$$

with $\beta_{\mu+1} \leq \beta_{\mu}$. Moreover, by noting the formula (see Gradshteyn and Ryzhik [7, equation 8.486.4])

$$
I_{\mu}^{\prime}(r)=\frac{\mu}{r} I_{\mu}(r)+I_{\mu+1}(r)
$$


we can obtain the following bounds

$$
\int_{0}^{R_{0}^{+}} r G_{i}^{+}(r) d r \leq C \mu_{i}^{+} e^{4 R_{0}^{+}}, \quad \int_{0}^{r_{0}^{+}} r G_{i}^{+}(r) d r \geq \delta_{0} \mu_{i}^{+} e^{-4 R_{0}^{+}}\left[\frac{r_{0}^{+}}{R_{0}^{+}}\right]^{2 \mu_{i}^{+}}
$$

with constants $0<\delta_{0}<C<\infty$. This yields

$$
\lambda_{\max }\left(F_{\bar{\Omega}^{+}}\right) \leq C \mu_{m}^{+}, \quad \lambda_{\min }\left(F_{\Omega^{+}}\right) \geq \delta_{0} \min \left[1, \mu_{m}^{+}\left(\frac{r_{0}^{+}}{R_{0}^{+}}\right)^{2 \mu_{m}^{+}}\right] .
$$

Similarly, we obtain

$$
\lambda_{\max }\left(F_{\bar{\Omega}^{-}}\right) \leq C \mu_{n}^{-}, \quad \lambda_{\min }\left(F_{\Omega^{-}}\right) \geq \delta_{0} \min \left[1, \mu_{n}^{-}\left(\frac{r_{0}^{-}}{R_{0}^{-}}\right)^{2 \mu_{n}^{-}}\right] .
$$

At least one of the following two inequalities

$$
r_{0}^{+}<R_{0}^{+}, \quad r_{0}^{-}<R_{0}^{-}
$$

must hold. Therefore,

$$
\min \left[\lambda_{\min }\left(F_{\underline{\Omega}^{+}}\right), \lambda_{\min }\left(F_{\underline{\Omega}^{-}}\right)\right]=\delta_{0} \min \left[\mu_{m}^{+}\left(\frac{r_{0}^{+}}{R_{0}^{+}}\right)^{2 \mu_{m}^{+}}, \mu_{n}^{-}\left(\frac{r_{0}^{-}}{R_{0}^{-}}\right)^{2 \mu_{n}^{-}}\right]
$$

for some large numbers $\mu_{m}^{+}$and $\mu_{n}^{-}$. Consequently, Theorem 4.1 yields

$$
\begin{aligned}
\text { cond } \leq & C t w\left[\left(1+p_{\max }\right) k_{m, n}+\frac{C_{s}}{w}\right]^{2} . \\
& \times\left[\frac{\max \left[\mu_{m}^{+}, \mu_{n}^{-}\right]}{\min \left\{\mu_{m}^{+}\left(r_{0}^{+} / R_{0}^{+}\right)^{2 \mu_{m}^{+}}, \mu_{n}^{-}\left(r_{0}^{-} / R_{0}^{-}\right)^{2 \mu_{n}^{-}}\right\}}\right]^{1 / 2} .
\end{aligned}
$$

The desired inequality (4.20) is obtained from the fact

$$
\mu_{m}^{+}=O\left(\mu_{m}^{-}\right)
$$

which results from $\mu_{i}^{ \pm}=i \pi / \Theta^{ \pm}$and the assumption $m=O(n)$. This completes the proof of Corollary 4.1 .

As a result of Corollary 4.1, the following formula holds for $w=1 / M$ :

$$
\text { cond }=O\left\{M \times \max \left[\left(\frac{R_{0}^{+}}{r_{0}^{+}}\right)^{\mu_{m}^{+}},\left(\frac{R_{0}^{-}}{r_{0}^{-}}\right)^{\mu_{n}^{-}}\right]\right\},
$$

provided that the bounds (3.14) are satisfied.

To end this section, we will make a few observations on how the shape of subdomains affects the stability of computations. If the solution domain is a rhomboid (Figure 2), the values of cond in Case b with a straight line $\Gamma_{0}$ are smaller than those in Case a with a circular arc $\Gamma_{0}$ (based on (4.20) or (4.32)), 


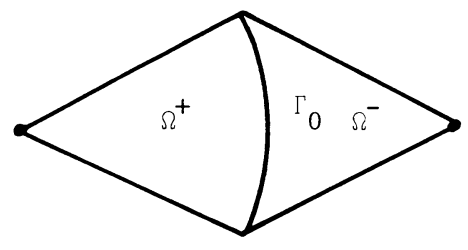

a

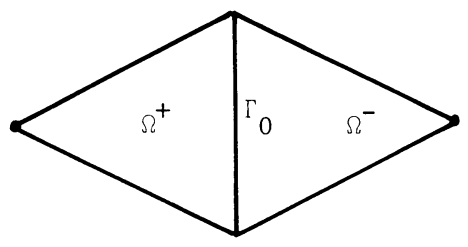

b

Figure 2. Partitions of a rhomboid

a) A circular arc $\Gamma_{0}$

b) A straight line $\Gamma_{0}$
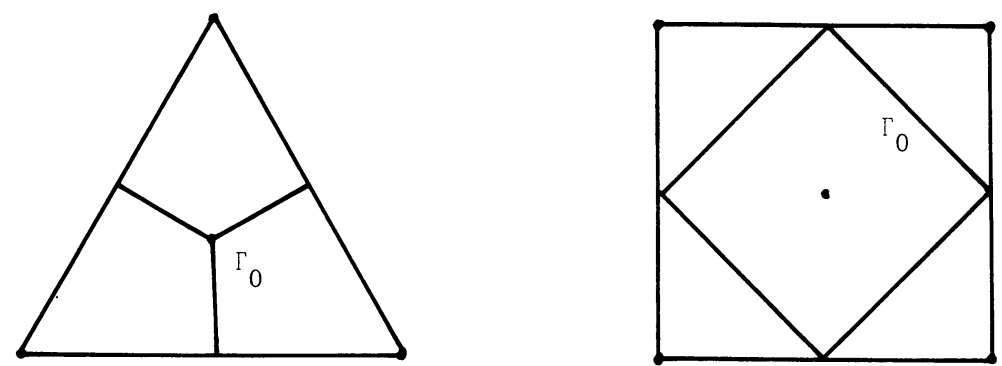

FIGURE 3. Good partitions for boundary methods

because in Case b, the maximum ratios $R_{0}^{+} / r_{0}^{+}$and $R_{0}^{-} / r_{0}^{-}$are closer to 1 . Also the divisions in Figure 3 should have good stability properties. These conclusions will be verified by numerical examples given in the next section.

\section{NUMERICAL EXPERIMENTS}

In this section, we will apply the boundary method to an interface problem and investigate the effect of different admissible functions and different divisions of the solution domain on the accuracy and stability of numerical solutions.

Consider the problem

$$
\begin{gathered}
p^{+}(-\Delta u+u)=0 \text { in } \Omega^{+}, \\
p^{-}(-\Delta u+u)=0 \text { in } \Omega^{-}, \\
u^{+}=u^{-}, \quad p^{+} u_{\nu}^{+}=p^{-} u_{\nu}^{-} \text {on } \bar{\Gamma}_{0}, \\
u=1 \quad \text { on } \partial \Omega,
\end{gathered}
$$

where $\Omega^{*}=\Omega^{+} \cup \Omega^{-}$(see Figure 4$), \Omega^{*}$ is a square domain $(-1<x<$ $1,-1<y<1), \Omega^{-}$is a smaller square domain $\left(-\frac{1}{2}<x<\frac{1}{2},-\frac{1}{2}<y<\frac{1}{2}\right)$, $\bar{\Gamma}_{0}$ is the interface boundary of $\Omega^{+}$and $\Omega^{-}$, and $p^{+}$and $p^{-}$are different positive constants. Because of the model's symmetry, it suffices to solve equations (5.1)-(5.4) only on $\Omega$, the eighth part of $\Omega^{*}$ as in Figure 5 .

We note that no numerical solution exists of such a complicated interface problem, while the infinite element approach of Han [8] and Thatcher $[19,20]$ is invalid for the equation $-\Delta u+u=0$. 


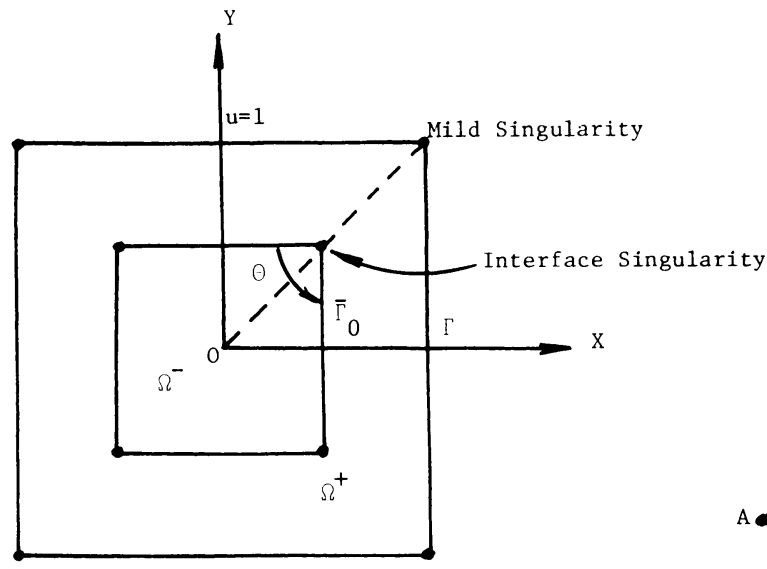

FIGURE 4. An interface problem on the domain $\Omega^{*}$

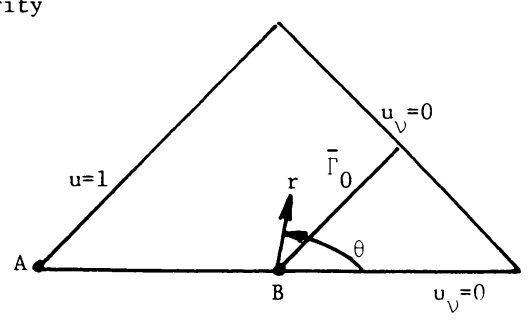

FIGURE 5. A fundamental region $\Omega$ of $\Omega^{*}$

Since the intersection angle of the interface is $\Theta=\pi / 2$, we will use the following admissible functions:

$$
v_{m}^{(0)}=\sum_{i=0}^{4 m-1} c_{i} \frac{I_{\mu_{i}}(r)}{I_{\mu_{i}}\left(\frac{1}{2}\right)} \boldsymbol{\Phi}_{\mu_{i}}(\theta),
$$

where $c_{i}$ are unknown coefficients, $I_{\mu}(r)$ are the Bessel functions for a purely imaginary argument, and $\Phi_{\mu_{i}}$ include both Kellogg eigenfunctions (Kellogg [911], also see Strang and Fix [18]) and additional eigenfunctions [12, 13] for a periodic Sturm-Liouville interface problem.

In fact, the Kellogg functions are defined by

$$
\boldsymbol{\Phi}_{\hat{\mu}_{j}}(\theta)=\left\{\begin{array}{l}
\cos \mu_{j} \theta, \quad|\theta|<\boldsymbol{\Theta} / 2, \\
\hat{\alpha}_{j} \cos \mu_{j}(\pi-\theta), \quad|\theta|>\boldsymbol{\Theta} / 2,
\end{array}\right.
$$

where the constants $\hat{\alpha}_{j}$ and $\mu_{j}$ satisfy

$$
\begin{gathered}
\hat{\alpha}_{j}=\frac{\cos \mu_{j} \Theta / 2}{\cos \mu_{j}(\pi-\Theta / 2)}, \\
p^{-} \tan \mu_{j} \Theta / 2+p^{+} \tan \mu_{j}(\pi-\Theta / 2)=0 .
\end{gathered}
$$

Take $\Theta=\pi / 2$ as an example; it follows from equations (5.6)-(5.8) that (see Strang and Fix [18, pp. 262])

$$
\mu_{j}=4 j \pm \alpha^{*},
$$

where the constant $\alpha^{*}$ is defined by

$$
\alpha^{*}=\frac{4}{\pi} \arctan \left(\frac{3+p^{-} / p^{+}}{1+3 p^{-} / p^{+}}\right)^{1 / 2} \text {. }
$$


It is pointed out in $[12,13]$ that when $\Theta=\pi / 2$ the Kellogg functions (5.6)(5.10). are not complete. The additional functions $\Phi_{\mu_{i}}(\theta)$ with $\mu_{i}=4 i$ and $4 i+2$ are provided as

$$
\Phi_{4 i}(\theta)=\cos 4 i \theta, \quad i=0,1, \ldots,
$$

and

$$
\Phi_{4 i+2}(\theta)=\left\{\begin{array}{lr}
\cos (4 i+2) \theta, & |\theta|<\Theta / 2=\pi / 4, \\
\frac{p^{-}}{p^{+}} \cos (4 i+2) \theta, & |\theta|>\pi / 4, \text { for } i=0,1, \ldots
\end{array}\right.
$$

Now both the Kellogg functions (5.6) and the additional functions (5.11) form a complete set of eigenfunctions of the Sturm-Liouville problem, which are used as admissible functions (5.5).

Clearly, the admissible functions (5.5) satisfy equations (5.1)-(5.3) exactly. Because there exists a mild singularity $A$ in Figure 5 (see $[12,15]$ ), it is necessary to employ piecewise particular functions. In other words, we have to divide $\Omega$ into two or more subdomains such that any one subdomain includes at most one singularity (see Figure 6 or 7). Besides, we are also interested in the artificial boundary $\Gamma_{0}$ which is made up of circular arcs or straight lines (like in most applications).

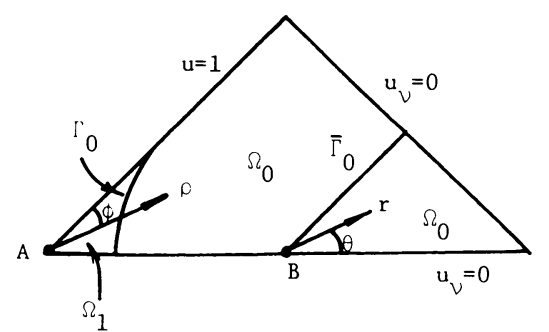

a

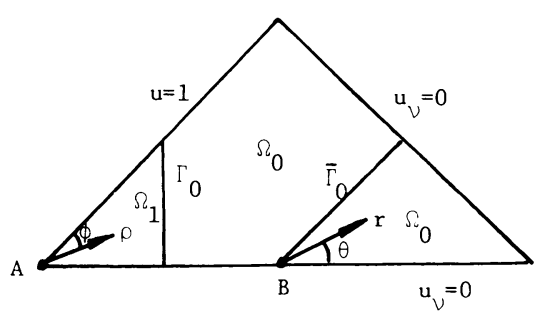

b

FIGURE 6. Partitions of $\Omega \subset \Omega^{*}$ into two subdomains $\Omega_{0}$ and $\Omega_{1}$
a) A circular arc $\Gamma_{0}$
b) A straight line $\Gamma_{0}$

On the basis of asymptotic expansions near the mild singularity $[12,15]$, we will choose the admissible functions

$$
v=\left\{\begin{aligned}
v_{m}^{(0)}= & \sum_{i=0}^{4 m-1} c_{i} \frac{I_{\mu_{i}}(r)}{I_{\mu_{i}}\left(\frac{1}{2}\right)} \Phi_{\mu_{i}}(\theta) \quad \text { in } \Omega_{0}, \\
v_{k}^{(1)}= & 1+\sum_{i=0}^{N_{E}} w_{2 i+1}^{*}(\rho) \sin 2(2 i+1) \phi \\
& +\sum_{i=0}^{k} d_{i} \frac{I_{2(2 i+1)}(\rho)}{I_{2(2 i+1)}\left(\frac{1}{2}\right)} \sin 2(2 i+1) \phi \text { in } \Omega_{1},
\end{aligned}\right.
$$


for the partitions in Figure 6, where $\Omega=\Omega_{0} \cup \Omega_{1}$, and choose

$$
v=\left\{\begin{aligned}
v_{m}^{(0)}= & \sum_{i=0}^{4 m-1} c_{i} \frac{I_{\mu_{i}}(r)}{I_{\mu_{i}}\left(\frac{1}{2}\right)} \Phi_{\mu_{i}}(\theta) \quad \text { in } \Omega_{0}, \\
v_{k}^{(1)}= & 1+\sum_{i=0}^{N_{E}} w_{2 i+1}^{*}(\rho) \sin 2(2 i+1) \phi \\
& +\sum_{i=0}^{k} d_{i} \frac{I_{2(2 i+1)}(\rho)}{I_{2(2 i+1)}\left(\frac{1}{2}\right)} \sin 2(2 i+1) \phi \quad \text { in } \Omega_{1}, \\
v_{n}^{(2)}= & \cosh (\xi \sin \omega)+\sum_{i=0}^{n} b_{i} \frac{I_{2 i+1}(\xi)}{I_{2 i+1}\left(\frac{1}{2}\right)} \sin (2 i+1) \omega \quad \text { in } \Omega_{2}, \\
v_{l}^{(3)}= & \sum_{i=0}^{l} a_{i} \frac{I_{2 i+1}(\eta)}{I_{2 i+1}\left(\frac{1}{2}\right)} \sin (2 i+1) \psi \quad \text { in } \Omega_{3},
\end{aligned}\right.
$$

for the partition in Figure 7, where $\Omega=\Omega_{0} \cup \Omega_{1} \cup \Omega_{2} \cup \Omega_{3}$, and the polar coordinates, $(r, \theta),(\rho, \phi),(\xi, w)$ and $(\eta, \psi)$, as shown in Figures 6 , 7. In (5.12) and (5.13), the constants $I_{\mu_{i}}\left(\frac{1}{2}\right)$ etc. in the denominators are used for scale factors.

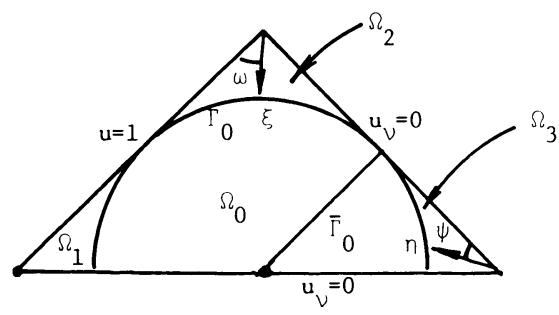

a

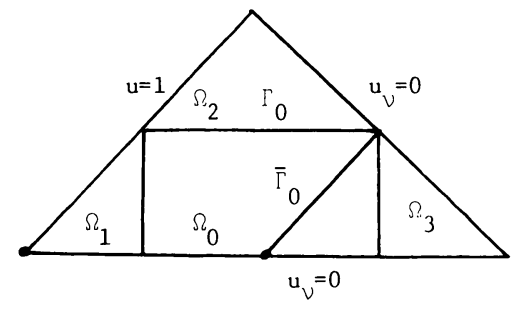

b

FIgURE 7. Partitions of $\Omega \subset \Omega^{*}$ into four subdomains $\Omega_{0}, \Omega_{1}, \Omega_{2}$ and $\Omega_{3}$

a) A semicircle $\Gamma_{0}$

b) Piecewise straight lines $\Gamma_{0}$

In (5.12) and (5.13), the known functions $w_{2 l+1}^{*}(\rho)$ are defined by

$$
w_{j}^{*}=w_{2 l+1}^{*}(\rho)=\sum_{i=0}^{\infty}\left(\alpha_{j, i}+\beta_{j, i} \ln \rho\right) \rho^{i+2}
$$

where $j=2 l+1$, and $\alpha_{j, i}$ and $\beta_{j, i}$ are real coefficients,

$$
\alpha_{j, 2 l+1}=\beta_{j, 2 l+1}=0 \text { for } l=0,1, \ldots,
$$


TABLE 1

Numerical results for partitions in Figure 6 with $w=1 / \max (4 m, 2(2 k+1))$

\begin{tabular}{|c|c|c|c|c|c|}
\hline Partition & $m$ & $k$ & $N_{E}$ & $|\varepsilon|_{B}$ & cond \\
\hline \multirow{4}{*}{$\begin{array}{c}\text { Partition } \\
\text { in } \\
\text { Figure 6a }\end{array}$} & 1 & 0 & 299 & $8.006 \times 10^{-3}$ & 38.3 \\
\cline { 2 - 6 } & 2 & 1 & 299 & $9.002 \times 10^{-5}$ & 219.7 \\
\cline { 2 - 6 } & 4 & 3 & 999 & $1.205 \times 10^{-6}$ & 24933 \\
\cline { 2 - 6 } & 5 & 4 & 999 & $2.299 \times 10^{-7}$ & 428726 \\
\cline { 2 - 6 } & 6 & 5 & 999 & $3.468 \times 10^{-8}$ & 7868170 \\
\hline \multirow{4}{*}{$\begin{array}{c}\text { Partition } \\
\text { in }\end{array}$} & 1 & 1 & 299 & $4.553 \times 10^{-3}$ & 48 \\
\cline { 2 - 6 } & 2 & 1 & 299 & $5.872 \times 10^{-5}$ & 214 \\
\cline { 2 - 6 } Figure 6b & 4 & 3 & 999 & $1.867 \times 10^{-7}$ & 4707 \\
\cline { 2 - 6 } & 5 & 4 & 999 & $3.126 \times 10^{-8}$ & 32564 \\
\cline { 2 - 6 } & 6 & 5 & 999 & $3.388 \times 10^{-9}$ & 291763 \\
\hline
\end{tabular}

and the coefficients $\alpha_{j, i}$ and $\beta_{j, i}$ are defined by the following recursive formulas $[12,15]$ :

1. When $(i+2)>2 j$,

$$
\begin{aligned}
\beta_{j, i} & =\frac{1}{(i+2)^{2}-(2 j)^{2}} \beta_{j, i-2}, \\
\alpha_{j, i} & =\frac{1}{(i+2)^{2}-(2 j)^{2}}\left[\alpha_{j, i-2}-2(i+2) \beta_{j, i}\right] .
\end{aligned}
$$

2. When $(i+2)<2 j$,

$$
\beta_{j, i}=0, \quad \alpha_{j, 0}=\frac{b_{j}}{4\left(1-j^{2}\right)}, \quad \alpha_{j, i}=\frac{\alpha_{j, i-2}}{(i+2)^{2}-4 j^{2}} \quad \text { for } i \geq 2 .
$$

3. When $i+2=2 j$,

$$
\alpha_{j, i}=0, \quad \beta_{1,0}=b_{1} / 4, \quad \beta_{j, i}=\frac{\alpha_{j, i-2}}{2(i+2)} \quad \text { for } i \geq 2
$$

where $b_{j}=4 / j \pi$.

Since the admissible functions (5.12)-(5.13) satisfy equations (5.1)-(5.4) and the symmetric boundary conditions $\partial u / \partial v=0$ on $\partial \Omega$ in Figure 5 , the boundary method (2.13) needs to approximate only on the artificial interface $\Gamma_{0}$.

In our calculation, $p^{-}=1$ and $p^{+}=0.2$; then $\alpha^{*}$ from $(5.10)$ has the value $\alpha^{*}=0.78365310406121$. The error norms and condition numbers of the numerical solutions are summarized in Tables 1 and 2. All results were 
TABLE 2

Numerical results for partitions in Figure 7

with $w=1 / \max (4 m, 2(2 k+1), 2 n+1,4 l)$

\begin{tabular}{|c|c|c|c|c|c|c|c|}
\hline Partition & $l$ & $m$ & $n$ & $k$ & $N_{E}$ & $|\varepsilon|_{B}$ & cond \\
\hline \multirow{6}{*}{$\begin{array}{l}\text { Partition } \\
\text { in } \\
\text { Figure } 7 \mathrm{a}\end{array}$} & 0 & 1 & 2 & 0 & 299 & $5.854 \times 10^{-3}$ & 70.5 \\
\hline & 1 & 2 & 3 & 1 & 299 & $8.105 \times 10^{-5}$ & 168.1 \\
\hline & 2 & 3 & 5 & 2 & 299 & $8.281 \times 10^{-6}$ & 319.4 \\
\hline & 3 & 4 & 7 & 3 & 999 & $6.951 \times 10^{-7}$ & 1587 \\
\hline & 4 & 5 & 9 & 4 & 999 & $9.425 \times 10^{-8}$ & 8201 \\
\hline & 5 & 6 & 11 & 5 & 999 & $1.730 \times 10^{-8}$ & 43976 \\
\hline \multirow{7}{*}{$\begin{array}{l}\text { Partition } \\
\text { in } \\
\text { Figure } 7 b\end{array}$} & 0 & 1 & 2 & 0 & 299 & $3.179 \times 10^{-3}$ & 48.1 \\
\hline & 1 & 2 & 3 & 1 & 299 & $8.715 \times 10^{-5}$ & 81.2 \\
\hline & 2 & 3 & 5 & 2 & 299 & $4.874 \times 10^{-6}$ & 118 \\
\hline & 3 & 4 & 7 & 3 & 999 & $3.184 \times 10^{-7}$ & 156 \\
\hline & 4 & 5 & 9 & 4 & 999 & $2.345 \times 10^{-8}$ & 228 \\
\hline & 5 & 6 & 11 & 5 & 999 & $2.164 \times 10^{-9}$ & 571 \\
\hline & 6 & 7 & 13 & 6 & 3299 & $2.075 \times 10^{-10}$ & 1443 \\
\hline
\end{tabular}

computed in double precision on an IBM 360 computer at the University of Toronto. The Bessel functions for a purely imaginary argument were evaluated by using a standard subroutine library.

From the numerical results listed in Tables 1 and 2, we can find the following asymptotic relations:
(5.19a) $|\varepsilon|_{B}=O\left(0.68^{M_{1}}\right)$,
cond $=O\left(1.79^{M_{1}}\right)$
for Figure 6a,
(5.19b) $|\varepsilon|_{B}=O\left(0.64^{M_{1}}\right)$,
cond $=O\left(1.55^{M_{1}}\right) \quad$ for Figure $6 \mathrm{~b}$,
(5.19c) $|\varepsilon|_{B}=O\left(0.81^{M_{2}}\right)$,
cond $=O\left(1.23^{M_{2}}\right) \quad$ for Figure $7 \mathrm{a}$,
(5.19d) $|\varepsilon|_{B}=O\left(0.74^{M_{2}}\right)$,
cond $=O\left(1.12^{M_{2}}\right)$
for Figure 7b,

where $M_{1}$ and $M_{2}$ are the total numbers of unknown coefficients, $M_{1}=4 \mathrm{~m}+$ $k+1$ and $M_{2}=4 m+k+n+l+3$. It is worth noting that the solution errors $|\varepsilon|_{B}$ in (5.19), as well as $\|\varepsilon\|_{H}$ by Corollary 3.1 , show the exponential convergence rates with respect to the total number of unknowns.

Traditional integration rules such as Simpson's rule can be used for computing the integrals in (3.9) as long as $m, k, n, l$ are not too large. We expect this to be the case in most practical applications. Otherwise, special treatments are needed for estimating the integration error in case of highly oscillatory integrands. Although $N_{E} \quad\left(i \leq N_{E}\right)$ is large, since the size of the coefficient functions $w_{2 i+1}^{*}(\rho)$ at $\sin (2 i+1) \phi$ is of the order $O\left(1 /(2 i+1)^{3}\right)$, we have obtained good accuracy using the Newton-Cotes formulas with appropriate subdomains. 
TABLE 3

Coefficients in (5.13) for the partitions in Figure $7 \mathrm{~b}$ with $l=6, m=7, n=13, k=6, N_{E}=3299$ and $w=1 / 28$

A. Coefficients $a_{i}$ and $d_{i}$

\begin{tabular}{|c|c|c|c|}
\hline$i$ & $a_{i}$ & $i$ & $d_{i}$ \\
\hline 0 & $+.6062843226890 \times 10^{0}$ & 0 & $-.1215834775893 \times 10^{0}$ \\
1 & $-.1613104434818 \times 10^{-2}$ & 1 & $+.1933144810146 \times 10^{-2}$ \\
2 & $+.1795608091823 \times 10^{-3}$ & 2 & $-.1784437702781 \times 10^{-3}$ \\
3 & $-.2122223809031 \times 10^{-4}$ & 3 & $+.2396263820099 \times 10^{-4}$ \\
4 & $+.3119724065311 \times 10^{-5}$ & 4 & $-.3744000695104 \times 10^{-5}$ \\
5 & $-.4978713964725 \times 10^{-6}$ & 5 & $+.6298085376459 \times 10^{-6}$ \\
6 & $+.6260235169312 \times 10^{-7}$ & 6 & $-.8328685743963 \times 10^{-7}$ \\
\hline
\end{tabular}

B. Coefficients $b_{i}$

\begin{tabular}{|c|c|c|c|}
\hline$i$ & $b_{i}$ & $i$ & $b_{i}$ \\
\hline 0 & $-.4954573232094 \times 10^{0}$ & 7 & $-.6376812990395 \times 10^{-5}$ \\
1 & $+.3113760526508 \times 10^{-1}$ & 8 & $-.5328917747106 \times 10^{-5}$ \\
2 & $+.3729769816638 \times 10^{-2}$ & 9 & $+.1038709724291 \times 10^{-5}$ \\
3 & $-.3495905082665 \times 10^{-3}$ & 10 & $+.8676086700602 \times 10^{-6}$ \\
4 & $-.2686360265942 \times 10^{-3}$ & 11 & $-.1755774436774 \times 10^{-6}$ \\
5 & $+.4539675028774 \times 10^{-4}$ & 12 & $-.1094832562485 \times 10^{-6}$ \\
6 & $+.3495651965563 \times 10^{-4}$ & 13 & $+.2276829890655 \times 10^{-7}$ \\
\hline
\end{tabular}

C. Coefficients $c_{i}$

\begin{tabular}{|c|c|c|c|c|c|}
\hline$i$ & $\mu_{i}$ & $c_{i}$ & $i$ & $\mu_{i}$ & $c_{i}$ \\
\hline 0 & 0 & $+.7006583869858 \times 10^{0}$ & 14 & 14 & $-.247584087615 \times 10^{-6}$ \\
1 & $\alpha^{*}$ & $-.1147786373534 \times 10^{0}$ & 15 & $16-\alpha^{*}$ & $+.2096278762216 \times 10^{-6}$ \\
2 & 2 & $-.1289426764916 \times 10^{-1}$ & 16 & 16 & $-.1639818111048 \times 10^{-6}$ \\
3 & $4-\alpha^{*}$ & $+.5778523669877 \times 10^{-2}$ & 17 & $16+\alpha^{*}$ & $+.8750969335584 \times 10^{-7}$ \\
4 & 4 & $-.2974571685497 \times 10^{-2}$ & 18 & 18 & $-.3299285102818 \times 10^{-7}$ \\
5 & $4+\alpha^{*}$ & $+.4847232200890 \times 10^{-3}$ & 19 & $20-\alpha^{*}$ & $+.2719051046239 \times 10^{-7}$ \\
6 & 6 & $-.3979913627169 \times 10^{-4}$ & 20 & 20 & $-.2200843353643 \times 10^{-7}$ \\
7 & $8-\alpha^{*}$ & $+.4066132127014 \times 10^{-4}$ & 21 & $20+\alpha^{*}$ & $+.1234412863930 \times 10^{-7}$ \\
8 & 8 & $-.1101625715174 \times 10^{-4}$ & 22 & 22 & $-.4196295750141 \times 10^{-8}$ \\
9 & $8+\alpha^{*}$ & $+.9399214433512 \times 10^{-5}$ & 23 & $24-\alpha^{*}$ & $+.6048883773878 \times 10^{-8}$ \\
10 & 10 & $-.4499904891205 \times 10^{-5}$ & 24 & 24 & $-.1820811027684 \times 10^{-8}$ \\
11 & $12-\alpha^{*}$ & $+.2410784033101 \times 10^{-5}$ & 25 & $24+\alpha^{*}$ & $+.1910127167080 \times 10^{-8}$ \\
12 & 12 & $-.2382249955680 \times 10^{-5}$ & 26 & 26 & $-.6138215056792 \times 10^{-9}$ \\
13 & $12+\alpha^{*}$ & $+.9508207885880 \times 10^{-6}$ & 27 & $28-\alpha^{*}$ & $+.1685056895614 \times 10^{-9}$ \\
\hline \multicolumn{6}{|c|}{} \\
\hline
\end{tabular}

We note that the related integration does not involve the matrix $B$, which is used to calculate the expansion coefficients.

First, let us compare the results in (5.19). The values of both $|\varepsilon|_{B}$ and cond for Figure $6 b$ (or $7 b$ ) are smaller than those for Figure 6a (or 7a). This agrees with the conclusions in $\S 4$ that using straight lines $\Gamma_{0}$ is more advantageous. 
Second, we note that the condition number behavior

$$
\text { cond }=O\left(1.12^{M_{2}}\right)
$$

for the subdomain of Figure $7 \mathrm{~b}$ is very good. This demonstrates the fact that approximate solutions calculated by using boundary methods can have excellent stability if the domain decomposition is equitable and if reasonable admissible functions are chosen analytically. It is because of this excellent stability and extremely high accuracy that boundary methods are very promising for solving certain partial differential equations in complicated domains, especially in the presence of singularities.

Third, the best results in our calculation,

$$
|\varepsilon|_{B}=2 \times 10^{-10}, \quad \text { cond }=1443 \text {, }
$$

correspond to 56 unknown coefficients, which are listed in Table 3. It follows from (5.21) and Corollary 3.1 that

$$
\|\varepsilon\|_{H}=O\left(10^{-9}\right) .
$$

Therefore, relative average errors of solutions and their generalized derivatives in $\Omega$, obtained by boundary methods, can achieve $O\left(10^{-9}\right)$ since $\|u\|_{H}=$ $O(1)$. This shows the extreme accuracy of our numerical solutions. On the other hand, the condition number is only 1443. Such a stability behavior can even cope with the stability problems arising in nonconforming combined methods [14].

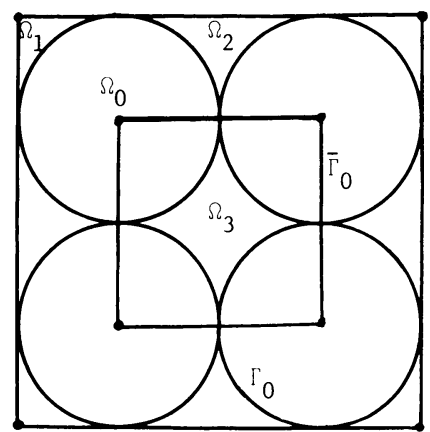

a

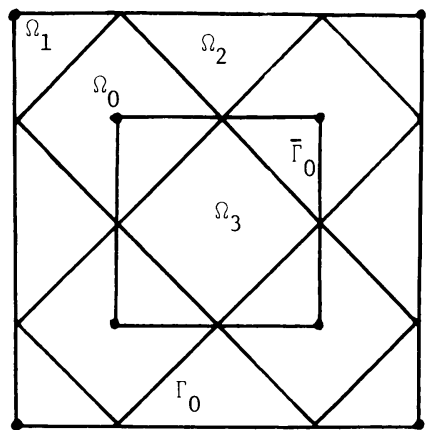

b

FIGURE 8 . The partitions of the whole solution domain $\Omega^{*}$, corresponding to Figure 7
a) Circles $\Gamma_{0}$
b) Piecewise straight lines $\Gamma_{0}$

In view of the above analysis and numerical experiments, we conclude that the best partition for our problem is that of Figure $7 b$ (i.e., Figure $8 b$ on the whole solution domain $\Omega^{*}$ ).

\section{ACKNOWLEDGMENTS}

This work was supported by the Departments of Computer Science, Mathematics and Applied Mathematics, University of Toronto, the Department of 
Computer Science, Concordia University, Centre de recherche informatique de Montreal, Inc., and in part by the Natural Science and Engineering Research Council of Canada under Grant No. A8651 and B94, by the Fonds pour la Formation de Chercheurs et l'Aide à la Recherche of Québec, and by the Ministère de l'Enseignement Supérieur et de la Science (Action Structurante). We also would like to thank the referee for his valuable suggestions.

\section{BIBLIOGRAPHY}

1. M. Abramowitz and I. A. Stegun, Handbook of mathematical functions with formulas, graphs and mathematical tables, Dover, New York, 1980.

2. A. K. Aziz, M. R. Dorr and R. B. Kellogg, A new approximation method for the Helmholtz equation in an exterior domain, SIAM J. Numer. Anal. 19 (1982), 899-908.

3. A. K. Aziz, R. B. Kellogg and A. B. Stephens, Least squares methods for elliptic systems, Math. Comp. 44 (1985), 53-70.

4. F. E. Browder, Approximation by solutions of partial differential equations, Amer. J. Math. 84 (1962), 134-160.

5. E. W. Cheney, Introduction to approximation theory, McGraw-Hill, New York, 1966.

6. S. C. Eisenstat, On the rate of convergence of the Bergman-Vekua method for the numerical solution of elliptic boundary value problems, SIAM J. Numer. Anal. 11 (1974), 654-680.

7. I. S. Gradshteyn and I. M. Ryzhik, Table of integrals, series, and products, Academic Press, New York, 1980.

8. H. Han, The numerical solution of interface problems in finite element methods, Numer. Math. 39 (1982), 39-50.

9. R. B. Kellogg, Singularities in interface problems, in Numerical Solution of Partial Differential Equations II (B. Hubbard, ed.), Academic Press, New York, 1971, pp. 351-400.

10. _ Higher order singularities for interface problems, in The Mathematical Foundations of the Finite Element Method with Application to Partial Differential Equations (A. K. Aziz, ed.). Academic Press, New York, London, 1972, pp. 589-602.

11. __, On the Poisson equations with intersecting interface, Applicable Anal. 4 (1975), 101-129.

12. Z. C. Li, Numerical methods for elliptic boundary value problems with singularities, Part I: Boundary methods for solving elliptic problems with singularities; Part II: Nonconforming combinations for solving elliptic problems with singularities, Ph.D. thesis, Department of Mathematics and Applied Mathematics, University of Toronto, May 1986.

13. __ A note on Kellogg's eigenfunctions of a periodic Sturm-Liouville system, Appl. Math. Letters 1 (1988), 123-126.

14. _ A nonconforming combination for elliptic problems with interfaces, J. Comput. Phys. 80 (1989), 288-313.

15. Z. C. Li and R. Mathon, Boundary approximation methods for solving elliptic problems on unbounded domains, J. Comput. Phys. (to appear).

16. Z. C. Li, R. Mathon and P. Sermer, Boundary methods for solving elliptic problems with singularities and interfaces, SIAM J. Numer. Anal. 24 (1987), 487-498.

17. S. L. Sobolev, Application of functional analysis in mathematical physics, Transl. Math. Monographs, vol. 7, Amer. Math. Soc., Providence, R.I., 1963.

18. G. Strang and G. J. Fix, An analysis of finite element methods, Prentice-Hall, Englewood Cliffs, N.J., 1973.

19. R. W. Thatcher, The use of infinite grid refinement at singularities in the solution of Laplace's equation, Numer. Math. 25 (1976), 163-178.

20. __ On the finite element method for unbounded regions, SIAM J. Numer. Anal. 15 (1978), 466-477. 
21. A. N. Tikhonov and A. A. Samarskii, Equations of mathematical physics, Macmillan, New York, 1973.

Centre de Recherche Informatique de Montreal, Inc., 1550 de Maisonneuve Blvd., West, Bureau 901, Montreal, Quebec H3G 1N2, Canada

Department of Computer Science, Concordia University, 1455 de Maisonneuve Blvd., West, Montreal, Quebec H3G IM8, Canada.

Department of Computer Science, University of Toronto, Toronto, Ontario M5S 1A4, CANADA 